\title{
Reflets
}

Revue ontaroise d'intervention sociale et communautaire

\section{Les intervenants et les organismes francophones en période de bouleversement}

\section{Anne Simard}

Volume 4, numéro 1, printemps 1998

Intervention en contextes minoritaires

URI : https://id.erudit.org/iderudit/026206ar

DOI : https://doi.org/10.7202/026206ar

Aller au sommaire du numéro

Éditeur(s)

Reflets : Revue ontaroise d'intervention sociale et communautaire

ISSN

1203-4576 (imprimé)

1712-8498 (numérique)

Découvrir la revue

Citer cet article

Simard, A. (1998). Les intervenants et les organismes francophones en période de bouleversement. Reflets, 4(1), 188-193. https://doi.org/10.7202/026206ar

Tous droits réservés (C) Reflets : Revue ontaroise d'intervention sociale et communautaire, 1998
Ce document est protégé par la loi sur le droit d'auteur. L'utilisation des services d'Érudit (y compris la reproduction) est assujettie à sa politique d'utilisation que vous pouvez consulter en ligne.

https://apropos.erudit.org/fr/usagers/politique-dutilisation/ 


\title{
Les intervenants et les organismes francophones en période de bouleversement
}

\author{
Anne Simard \\ Centre ontarien d'information en prévention, Toronto.
}

\section{Introduction}

Une panoplie de changements se vit actuellement (et depuis quelques temps) en Ontario dans les secteurs de la santé, de l'éducation et des services sociaux. Ces changements se font sentir aux niveaux provincial, local et communautaire. Bien qu'ils touchent tous les organismes et les intervenants dans ces secteurs, les services en français risquent d'être plus fortement touchés puisqu'ils sont moins nombreux et encore en voie de développement.

Une étude menée auprès des intervenants francophones oeuvrant en promotion de la santé en Ontario au printemps 1997 démontre que les coupures budgétaires affectent profondément leurs organismes et leur travail. Les coupures amènent des changements importants dont les répercussions sur le plan organisationnel sont la restructuration des services et la réduction des activités, notamment des services en français. Ils se font sentir au niveau du fonctionnement des professionnels également; ceuxci notent des changements dans leur état d'esprit, dans leurs conditions de travail et dans leurs priorités de travail. Cet article 
décrit l'impact du changement sur les intervenants francophones en promotion de la santé ainsi que les obstacles auxquels ils font face. L'étude comme telle comprend également une analyse de leurs besoins émergents et des moyens par lesquels les centres de ressources provinciaux en promotion de la santé peuvent mieux répondre aux besoins.

\section{Contexte}

Le Centre ontarien d'information en prévention (COIP) dessert les Franco-Ontariens depuis sept ans, offrant des services d'information, de formation, de consultation et de réseautage dans le domaine de la promotion de la santé. Ces services en français ont évolué selon les demandes et les besoins émergents de ses clients. Depuis deux ans, on ressentait un besoin de revoir les services et de vérifier s'ils répondaient toujours aux besoins des Franco-Ontariens et des intervenants qui travaillent auprès des communautés francophones. De plus, le COIP venait d'établir un partenariat avec le Centre for Health Promotion (CHP) de l'université de Toronto qui, lui aussi, voulait examiner le bienfondé d'offrir des services en français.

C'est ainsi que le COIP et le CHP ont entrepris conjointement une étude de besoins auprès des intervenants francophones oeuvrant en promotion de la santé à travers la province de l'Ontario.

\section{Les activités de recherche}

Afin de saisir l'ampleur et les répercussions du changement dans les secteurs de la santé, de l'éducation et des services sociaux, l'étude visait des intervenants francophones ou encore, les intervenants oeuvrant auprès de la communauté franco- 
ontarienne. Un questionnaire a été élaboré par une experte-conseil avec l'aide d'un comité consultatif.Un échantillon de 491 personnes a été sélectionné à partir de la banque de données du COIP, des unités et membres affiliés du CHP, des regroupements membres du RIFSSSO, des coalitions anti-tabac, et d'autres organismes pertinents. L'échantillon avait une représentation régionale ainsi qu'une représentation des différents milieux : les associations, les centres de santé communautaire, les organismes de services sociaux, les écoles, les bureaux de santé publique et les coalitions communautaires, parmi d'autres. Tous les individus faisant partie de l'échantillon ont reçu le questionnaire par la poste, suivi d'un rappel après deux semaines et d'un dernier rappel un mois après l'envoi.

En tout, 111 personnes ont répondu au questionnaire, soit un retour de 23 pour cent. La majorité d'entre elles ont le français comme langue maternelle (92 pour cent). Cependant, elles ne travaillent pas uniquement en français; la plupart travaillent dans les deux langues officielles (56 \%), $10 \%$ parlent l'anglais au bureau et $34 \%$ travaillent en français.

Environ $50 \%$ travaillent soit dans un bureau de santé publique (12\%), un centre de santé communautaire (10\%), un hôpital $(10 \%)$ ou un organisme de services sociaux (11\%). Il y a également des répondants qui proviennent du milieu scolaire (6\%), universitaire $(9 \%)$ et gouvernemental $(4 \%)$.

\section{L'impact du changement}

Les répondants sont profondément affectés par le changement: $90 \%$ ont indiqué que les programmes et les services qu'offrent leurs organismes changent en raison des bouleversements. Ce ne sont pas des changements faciles. Ils obligent la restructuration des services (chez $69 \%$ des répondants) ainsi que la réduction des activités et le fusionnement des services et programmes. Comme dit un répondant: "C'est une réorganisation totale du fonctionnement». 
Somme toute, les répondants révèlent que l'omniprésence du changement a un impact négatif sur leur organisme et leur communauté. Souvent la restructuration de l'organisme entraîne une réduction des activités et le fusionnement des programmes.

Les coupures semblent créer un cycle perturbant : il y a moins de services disponibles dans la communauté, les services qui existent toujours sont réduits (moins d'heures d'ouverture, moins de personnel) et les intervenants travaillent auprès d'une clientèle avec de plus grands besoins.

Les services en français ne sont pas nécessairement protégés lors de ces compressions, comme l'indique une répondante: «La restructuration de l'organisme se déroule au détriment des francophones». En effet, selon les résultats du sondage, la restructuration semble toucher particulièrement les services en français; il y a une réduction du soutien apporté aux services et aux programmes de langue française et des ressources en français, y compris les ressources humaines, matérielles et financières. Certains répondants affirment que le fait de vivre dans une région majoritairement francophone protège les services en français. Cependant, d'autres répondants de la même région constatent qu'il n'existe aucune protection.

Pour les intervenants eux-mêmes, le travail ne fait que croître. Selon $79 \%$ des répondants, le nombre de tâches à accomplir ne cesse d'augmenter. Leurs nouvelles tâches comprennent l'établissement de nouveaux partenaires et la recherche d'autres sources de financement. Leurs méthodes de travail doivent changer; ils notent un besoin accru de méthodes novatrices d'intervention: «Nous devons être créatifs car les anciennes méthodes sont hors d'usage». On ne peut plus se fier au gouvernement pour fournir les fonds nécessaires; celui-ci réduit ses contributions et les organismes coupent les services en conséquence. Les intervenants se disent obligés de faire appel à de nouvelles connaissances telles l'informatique, le marketing et l'autofinancement, afin de poursuivre leurs objectifs.

Les intervenants racontent que tout n'est pas perdu: «Les services en français se poursuivent, mais il faut vraiment se battre 
pour les conserver. Les gens se rallient et tentent de travailler plus ensemble». Il y a donc une nouvelle façon de travailler qui émerge, dans laquelle les intervenants se fient davantage au soutien des clients et des communautés ainsi que à l'appui des collègues et des organismes du voisinage. La collaboration et l'engagement $\mathrm{du}$ public et du personnel sont maintenant essentiels au bon déroulement et peut-être au maintien des services.

En effet, ils semblent chercher des moyens pour faire plus avec moins de ressources (y compris le temps.) Tous ces bouleversements affectent l'état d'esprit des intervenants qui se sentent surchargés : $58 \%$ disent qu'ils connaissent une baisse de moral et une perte d'enthousiasme pour leur travail. Selon une répondante: "Le personnel est stressé et déçu». Ceci n'est pas surprenant puisque certains disent qu'ils ressentent «la crainte de perdre leurs emplois» et "l'inquiétude face à l'avenir même des services".

Les coupures budgétaires créent des obstacles pour les intervenants et pour le bon déroulement de leur travail. Avec moins de ressources, il y a une réduction des adhésions aux organismes de soutien ou professionnels et moins de développement professionnel. On indique qu'il y a un manque de formation en français. De plus, $36 \%$ des répondants croient que leur organisme offre peu de soutien à la formation en français même si celle-ci est disponible.

Les Franco-Ontariens et les communautés francophones subissent également les séquelles de ces bouleversements. Puisque les organismes qui les desservent réduisent leurs services, les programmes en français deviennent moins accessibles. De plus, les commentaires des répondants mettent l'accent sur la «quasi impossibilité de démarrer les services en français», ce qui empêche l'épanouissement de la communauté francophone en Ontario puisque ces fonds de démarrage servait à «créer plus de services pour les francophones et une communauté francophone plus solide». 


\section{Conclusion}

Depuis 1995, les organismes et les intervenants dans les secteurs de la santé, de l'éducation et des services sociaux vivent une nouvelle réalité. Ils font face aux coupures et aux compressions budgétaires tout en essayant de continuer à desservir les communautés francophones de l'Ontario. Les répondants de la présente étude démontrent des capacités d'adaptation aux changements, tout en reconnaissant que ceux-ci obligent la mise en oeuvre des nouvelles façons de travailler et créent des besoins à combler pour les professionnels, les organismes et les communautés francophones à travers la province. 